\title{
Torsional saccadic oscillations
}

\author{
Marcello Cherchi* \\ Ken and Ruth Davee Department of Neurology, Northwestern Feinberg School of Medicine, Chicago, USA
}

\begin{abstract}
We present a case of torsional saccadic oscillations and discuss the possible underlying neuroanatomical substrate.
\end{abstract}

\section{Case report}

A 31-year-old man presented with a complaint of imbalance following head trauma. During childhood he had strabismus and "nystagmus" about which no further information was available. At age 29 he sustained head trauma during an assault. After the head trauma he began experiencing headaches, amnesia and episodes of tilting or rocking lightheadedness associated with blurry vision, oscillopsia and nausea (Table 1). The episodes were sometimes triggered by complex visual stimuli (such as following a rapidly moving target) and by changes in position of the head, and could range in duration from hours to days. The symptoms improved over several months, but worsened again after he fell 15 feet from a roof and landed on his back. The symptoms were not alleviated by baclofen, gabapentin, paroxetine or duloxetine. He denied any tinnitus, noise sensitivity, aural fullness, otalgia and hearing loss. Audiometry, otoacoustic emissions and vestibular evoked myogenic potentials were normal. MRI of the cervical spine was normal. MRI of the brain was reported as normal.

Video Frenzel oculography was remarkable for modest up beating nystagmus and an approximately $8 \mathrm{~Hz}$ pendular torsional oscillation that may be described as torsional flutter or torsional shimmer that was persistent in all directions of gaze and in all positions, unchanged by any maneuvers (Figure 1 and Video). The patient was lost to follow-up.

We are not aware of any prior reports of torsional saccadic oscillations. Beyond documenting it, we propose here some ideas regarding the possible underlying neuroanatomy. However, we begin the discussion with the neuroanatomy underlying horizontal saccades, and the possible mechanisms underlying horizontal saccadic oscillations, as these are better understood than their vertical and torsional counterparts.

\section{Horizontal saccades and the possible mechanisms of oscillations}

The brainstem nuclei known to be involved in generating horizontal saccades are the paramedian pontine reticular formation

Table 1. Symptoms at presentation in the patient with torsional saccadic oscillations.

\section{Oscillopsia}

Lightheadedness

Nausea

Headaches

Sensitivity to complex visual stimuli
[PPRF] (containing the burst neurons that generate the "pulse" signal needed to initiate a saccade), another area in the pontine reticular formation (containing the neurons that generate the "step" signal needed to maintain the eccentric position) and the pontine nucleus raphe interpositus [RIP] (containing the omnipause neurons that inhibit, or "gate," the burst neurons) [1-3].

Zee and Robinson [4] proposed a model to account for horizontal saccades and for the quick phase of horizontal nystagmus. This model began with the generally accepted concept that these fast eye movements are created by a "pulse-step" mechanism. The "pulse" component is phasic and encodes eye velocity; it is thought to arise from burst neurons located in the pontine paramedian reticular formation that are normally silent but fire rapidly immediately before and during the eye movement. The "step" component is tonic and encodes eye position; it is thought to arise from other neurons in the pontine reticular formation that integrate (in the mathematical sense) $[5,6]$ the velocity information from the immediately preceding "pulse" component in order to generate a position-coded signal to maintain the eye in the new, eccentric position by overcoming elastic restorative forces of the orbit [7].

Early investigators believed that the pulse was preprogrammed or

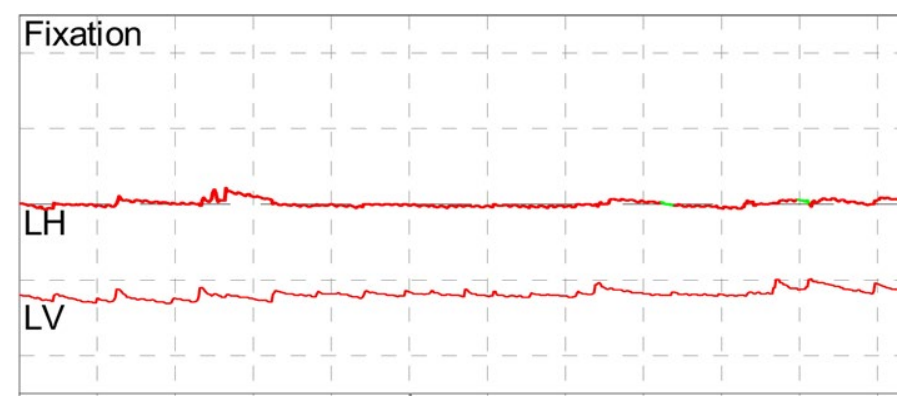

Figure 1. Videonystagmography. The top tracing reflects horizontal eye movements, the bottom tracing reflects vertical eye movements. Torsional eye movements are not directly measured, but are nevertheless manifest in the subtle horizontal oscillatory eye movement

Correspondence to: Marcello Cherchi, M.D., Ph.D., Ken and Ruth Davee Department of Neurology, Northwestern Feinberg School of Medicine, Abbott Hall, 11th Floor, 710 N. Lake Shore Drive, Chicago, IL 60611-3078, USA, Tel: 312-908-8266; E-mail: m-cherchi2@md.northwestern.edu

Received: April 26, 2015; Accepted: May 29, 2015; Published: June 01, 2015 


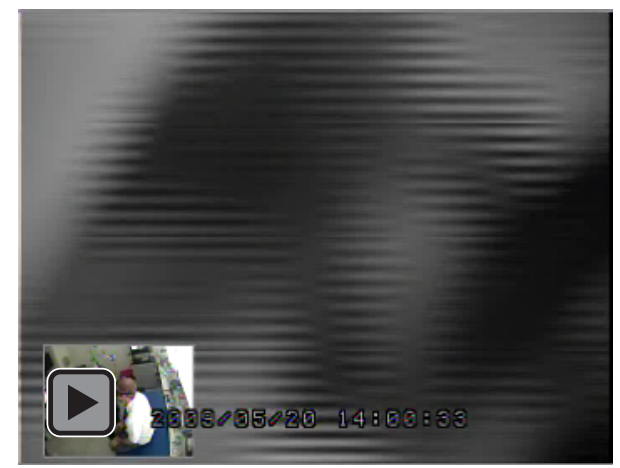

Video: The video shows infrared video oculography of torsional saccadic oscillation.

"ballistic" and, once initiated, could not be changed. However, Zee and Robinson made the key observation that patients with spinocerebellar degeneration had slow saccades that could be modified "in-flight", and therefore proposed that the "central nervous system generates saccades by driving the eyes to a calculated position in the orbit rather than preprogramming a neural pulse of a specified intensity and duration." The model they proposed for this involved two components: "(1) the neural commands coming to the saccadic pulse generator are coded to specify the final position in the orbit to which the eye must be brought rather than a preprogrammed distance the eye must be moved, and (2) the eye is automatically brought to its final position by a local pontine feedback loop that drives the eye until instantaneous and desired eye position are equal." They cited animal experiments that revealed a group of neurons in the pontine midline called "pause neurons" (elsewhere called "omnipause neurons" [OPN] that are located in the pontine nucleus raphe interpositus [RIP] [8]) that are silent while a saccade is in progress and resume firing when the saccade is complete; when these pause neurons are firing, they suppress saccades by inhibiting the burst neurons (that generate the pulse). In order for a saccade to initiate and proceed, these pause neurons must be inhibited in order to "release" (disinhibit) the burst neurons; once a saccade is in progress, the activity of the burst neurons inhibits the pause neurons. They postulate a "pathway that allows the output of the burst cells to feed back upon and inhibit the pause cells, which keeps the pause cells off as long as the saccade is in progress," and they describe this mutually inhibitory arrangement between burst neurons and pause neurons as a "latch" mechanism. This latch facilitates a saccade when there is a difference between the eye's instantaneous position and the eye's desired position (i.e., when the saccade is incomplete).

The neural substrate of this "latch" is unknown. However, simulation of this model, "revealed that the saccadic pulse generator circuit is inherently unstable and under appropriate conditions will cause the eyes to oscillate at a high frequency." As a potential mechanism for this instability, Zee and Robinson suggested, "one can hypothesize that the saccadic pulse generator [...] is inherently unstable because of a small delay in the eye position feedback loop." They later pointed out that this is fundamentally a "negative feedback model" [8].

The "delay in the negative feedback loop controlling saccade amplitude to produce saccadic oscillations" was later hypothesized to "pass through the cerebellar fastigial nuclei [...] and around the brainstem saccade generator," and this would predict that bilateral dysfunction of the cerebellar fastigial nuclei should eliminate the possibility of saccadic oscillation. However, the subsequent observation that a patient with bilateral cerebellar lesions of the cerebellar fastigial nuclei still could exhibit saccadic oscillations led Ramat et al. [8] to, "propose another model that accounts for saccadic oscillations based on 1) coupling of excitatory and inhibitory burst neurons in the brainstem and 2) the hypothesis that burst neurons show postinhibitory rebound discharge." In essence, this model, "is based on the positive feedback loops that are intrinsic in the brainstem connectivity of excitatory and inhibitory burst neurons" [8]. Specifically, this model "distributes the role of the burst neurons across two constituent types: excitatory (EBN) and inhibitory (IBN), which are connected across the midline $[\ldots]$ producing two positive feedback loops. These positive feedback connections provide another source of instability and lead to an alternative model for saccadic oscillations" [8]. This is crucially different from the earlier explanation [4] that involved a "negative feedback model." Ramat et al. [9] further developed the idea of the "post inhibitory rebound" (PIR) mechanism with additional support from observations of certain characteristics of saccadic oscillations in normal subjects, from a patient with microsaccadic oscillations and limb tremor, and from a patient with a surgical lesion of the fastigial nucleus. They also drew on research suggesting that the role of omnipause neurons is not simply complete inhibition of burst neurons, but rather modulation of the burst neuron activity, and that dysfunction of the omnipause neurons is the main cause of instability responsible for saccadic oscillations.

A review of the reciprocal influence between clinical observations and the theoretical modeling of saccades is presented in Ramat et al. [10].

\section{Torsional saccades and the possible substrate of oscillations}

The brainstem nuclei involved in generation of vertical and torsional eye movements are the nucleus raphe interpositus [RIP] (containing omnipause neurons), the rostral interstitial nucleus of the medial longitudinal fasciculus [riMLF] (containing burst neurons) and the interstitial nucleus of Cajal [INC] which animal experiments [11] suggest contain the integrator neurons for these movements.

Single-neuron recordings in monkeys [12] have identified burst neurons in the rostral interstitial nucleus of the median longitudinal fasciculus that generate ipsitorsional eye movements. Other experiments show that neurons in the interstitial nucleus of Cajal [INC] contain the integrator neurons not only for horizontal eye movements, but also for vertical and torsional eye movements [13].

The neuroanatomical substrate for vertical and torsional saccadic oscillations has been less thoroughly explored than that of horizontal saccadic oscillations. Shaikh et al. [14], based on a case of adult-onset Still's disease, postulate dysfunction in the interstitial nucleus of Cajal. In contrast, Washio et al. [15], based on a case of a cavernous angioma in the medial dorsal pons, speculate that combined lesions of the pontine nucleus raphe interpositus [RIP] and paramedian tracts [PMT] of cell groups "lying closely lateral to RIP" whose function is to "[relay] efference copy signals to the cerebellar floccular region [which] is important in maintenance of fixation."

Given the clinical observations regarding horizontal saccadic oscillations that prompted the evolution of the theoretical models from that presented by Zee and Robinson [4] to that offered by Ramat et al. [9], we find the concept of "post inhibitory rebound" discharge (resulting from the inherent instability of the interplay between two positive feedback loops, each of which involves a small delay) the more physiologically plausible explanation. If correct, this would favor the interstitial nucleus of Cajal as the likely neuroanatomical substrate, with torsional flutter arising from dysfunction of the nuclei themselves, 
or dysfunction of the reciprocal (commissural) connections between their presumed excitatory burst neurons and inhibitory burst neurons. For now this idea remains theoretical because Leigh and Zee [16] note that studies in humans of focal lesions of the interstitial nucleus of Cajal are lacking; and although the afferent and efferent projections between the interstitial nucleus of Cajal and vestibular and cochlear nuclei have been studied, the presumed commissural connections between the interstitial nuclei of Cajal have not. Nevertheless, if correct, this idea would predict that lesions in the ventral mesencephalic periaqueductal gray (such as anatomical distortion by hydrocephalus or a lesion in the floor of the fourth ventricle) that disrupt the presumed commissural connections between the interstitial nuclei of Cajal could result in torsional flutter.

\section{References}

1. Sparks DL (2002) The brainstem control of saccadic eye movements. Nat Rev Neurosci 3: 952-964. [Crossref]

2. Van Gisbergen JA, Robinson DA, Gielen S (1981) A quantitative analysis of generation of saccadic eye movements by burst neurons. J Neurophysiol 45: 417-442. [Crossref]

3. Fuchs AF, Kaneko CR, Scudder CA (1985) Brainstem control of saccadic eye movements. Annu Rev Neurosci 8: 307-337. [Crossref]

4. Zee DS, Robinson DA (1979) A hypothetical explanation of saccadic oscillations. Ann Neurol 5: 405-414. [Crossref]

5. Fukushima K, Kaneko CR, Fuchs AF (1992) The neuronal substrate of integration in the oculomotor system. Prog Neurobiol 39: 609-639. [Crossref]
6. Cannon SC, Robinson DA (1985) An improved neural-network model for the neural integrator of the oculomotor system: more realistic neuron behavior. Biol Cybern 53 93-108. [Crossref]

7. Robinson DA, Keller EL (1972) The behavior of eye movement motoneurons in the alert monkey. Bibl Ophthalmol 82: 7-16. [Crossref]

8. Ramat S, Leigh RJ, Zee DS, Optican LM (2005) Ocular oscillations generated by coupling of brainstem excitatory and inhibitory saccadic burst neurons. Exp Brain Res 160: 89-106. [Crossref]

9. Ramat S, Leigh RJ, Zee DS, Shaikh AG, Optican LM (2008) Applying saccade models to account for oscillations. Prog Brain Res 171: 123-130. [Crossref]

10. Ramat S, Leigh RJ, Zee DS, Optican LM (2007) What clinical disorders tell us about the neural control of saccadic eye movements. Brain 130: 10-35. [Crossref]

11. King WM, Fuchs AF, Magnin M (1981) Vertical eye movement-related responses of neurons in midbrain near intestinal nucleus of Cajal. J Neurophysiol 46: 549-562. [Crossref]

12. Hepp K, Vilis T, Henn V (1988) On the generation of rapid eye movements in three dimensions. Ann N Y Acad Sci 545: 140-153. [Crossref]

13. Crawford JD, Cadera W, Vilis T (1991) Generation of torsional and vertical eye position signals by the interstitial nucleus of Cajal. Science 252: 1551-1553. [Crossref]

14. Shaikh AG, Hain TC, Zee DS (2010) Oculomotor disorders in adult-onset Still's disease. J Neurol 257: 136-138. [Crossref]

15. Washio N, Suzuki Y, Yamaki T, Kase M, Ohtsuka K (2005) Vertical-torsional oscillations and dissociated bilateral horizontal gaze palsy in a patient with a pontine cavernous angioma. J Neurol Neurosurg Psychiatry 76: 283-285. [Crossref]

16. Leigh RJ, Zee DS (2006) The Neurology of Eye Movements, (4thedn), New York: Oxford University Press.

Copyright: $\left({ }^{2} 2015\right.$ Cherchi M. This is an open-access article distributed under the terms of the Creative Commons Attribution License, which permits unrestricted use, distribution, and reproduction in any medium, provided the original author and source are credited. 